\title{
Breakdown behavior of electronics at variable pulse repetition rates
}

\author{
S. Korte and H. Garbe \\ Institute of Electrical Engineering and Measurement Science, University of Hannover, Germany
}

\begin{abstract}
The breakdown behavior of electronics exposed to single transient electromagnetic pulses is subject of investigations for several years. State-of-the-art pulse generators additionally provide the possibility to generate pulse sequences with variable pulse repetition rate. In this article the influence of this repetition rate variation on the breakdown behavior of electronic systems is described. For this purpose microcontroller systems are examined during line-led exposure to pulses with repetition rates between $1 \mathrm{KHz}$ and $100 \mathrm{KHz}$. Special attention is given to breakdown thresholds and breakdown probabilities of the electronic devices.
\end{abstract}

\section{Introduction}

As mentioned above several investigations concerning the breakdown and destruction behavior of electronics during single pulse impact have been performed. In this context failure rates for destruction and breakdown phenomena have been defined (Camp et al., 2002). As this investigation focuses on the breakdown effects without destructing the devices the Breakdown Failure Rate (BFR) is an interesting quantity to be measured. For single pulse measurement it is defined as:

$\mathrm{BFR}=\frac{\text { number of breakdowns }}{\text { number of pulses }}$.

This definition gives a quantity for the breakdown probability of the electronic devices during single pulse measurements.

Pulse sequences consist of a lot of pulses, so this fact will cause very small BFR. Therefore it makes more sense to relate the number of breakdowns to the number of tested devices. The definition of Eq. (1) been modified as shown in Eq. (2).

$\mathrm{BFR}=\frac{\text { number of breakdowns }}{\text { total number of tested devices }}$

Correspondence to: S. Korte

(korte@ieee.org)
Equation (2) is similar to the general definition of a probability. With this definition a qualitative analysis of the breakdown behavior can also be performed with smaller test series.

The characteristic of a BFR with increasing amplitude of the disturbance signal can be approximated with the probability mass function of a Weibull Distribution (Camp, 2004). In order to get a more specified description of the breakdown behavior two variables have been defined (Camp et al., 2002). The Breakdown Threshold (BT) is the amplitude where $5 \%$ of the electronic devices break down. The Breakdown Bandwidth (BB) is the difference between the amplitudes where the BFR reaches 95 and 5\%.

Both variables provide the opportunity to get a quick comparison between different measurements.

In investigations with single pulses the following results where gained:

- BFR is increasing from 0 to 1 with increasing pulse amplitude (Camp, 2004)

- both BT and BB depend on the function of the investigated pin of the Equipment Under Test (EUT) (Camp, 2004)

- critical system states can be observed where the electronic system is highly vulnerable (Camp et al., 2004)

\section{Measurement setup}

In order to apply line-led disturbing signals to electronic devices a special measurement setup has been constructed in the past. Details of the microcontroller test circuit are shown in Fig. 1. It consists of a microcontroller PCB connected on both sides to a load which is constructed out of LEDs and resistors. With mounted chip this circuitry is able to run test programs which trigger the LEDs frequently to monitor the accurate function or a breakdown of the chips. Between

Published by Copernicus GmbH on behalf of the URSI Landesausschuss in der Bundesrepublik Deutschland e.V. 


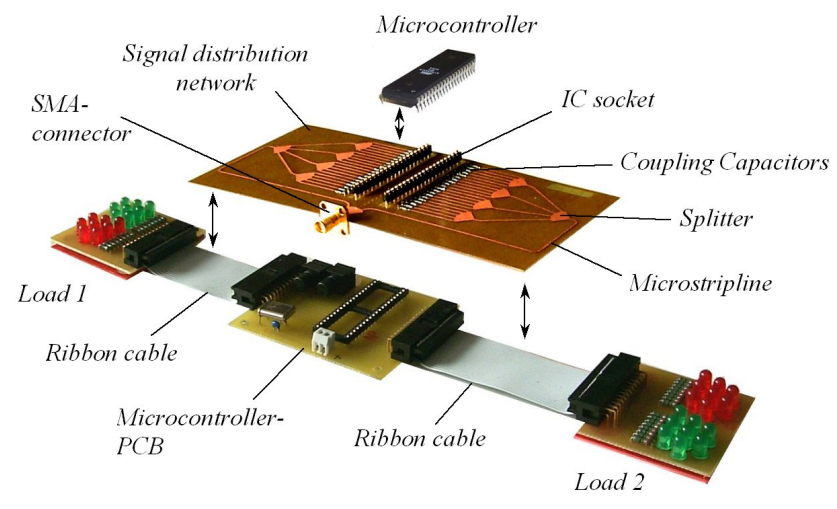

Fig. 1. Microcontroller Test Setup (Details).

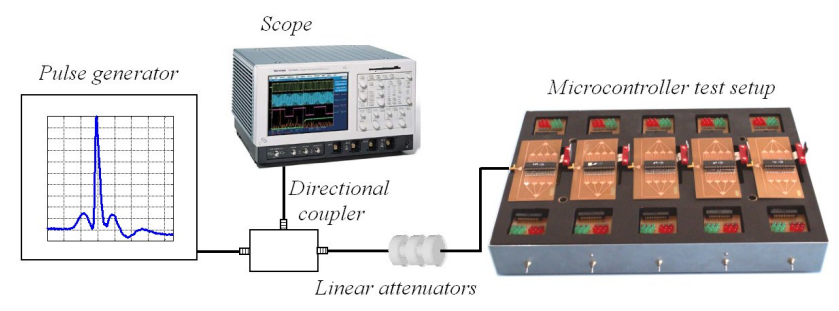

Fig. 2. Measurement setup (principle).

microcontroller and microcontroller PCB several signal distribution networks can be positioned. This networks have SMA connectors to connect arbitrary signal sources. Via microstriplines and coupling capacitors the signal is distributed to the considered pins. All other pins are terminated with $50 \mathrm{Ohm}$.

This circuitry is embedded in a microcontroller test setup which provides the possibility to apply disturbing signals line-led to several pins of the electronic devices in order to get information about the correlation between the function of the exposed pin and the breakdown behavior. The following pins are exposed:

- Supply pins

- Reset pin

- Clock pins

- I/O pins

As drafted in Fig. 2 a pulse generator is feeding via a directional coupler and several linear attenuators the test setup described above. Pulse shape and amplitude can be monitored with the scope connected to the directional coupler. The measurement setup is fed with pulse generators from Rheinmetall Waffe Munition GmbH. Rise time $t_{r}$, full width half max value $t_{\text {fwhm }}$ and possible repetition rates $f_{\text {rep }}$ of the pulse generators are shown in Table 1 .

Generator 1 was used to generate pulses with repetition rates $f_{\text {rep }}=1 \mathrm{kHz}$ and $f_{\text {rep }}=10 \mathrm{kHz}$ while Generator 2 pro-
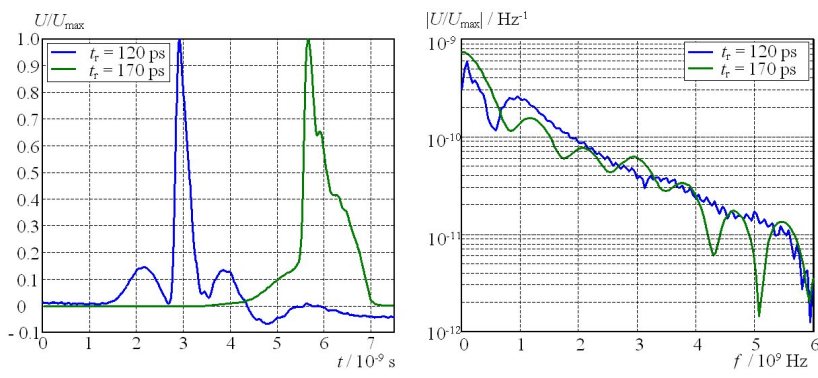

Fig. 3. Applied pulses in the time and frequency domains.

Table 1. Pulse generators - characteristics.

\begin{tabular}{cccc}
\hline Generator & $t_{r}$ & $t_{\text {fwhm }}$ & $f_{\text {rep }}$ \\
\hline 1 & $170 \mathrm{ps}$ & $500 \mathrm{ps}$ & $1 \mathrm{KHz}, 10 \mathrm{KHz}$ \\
\hline 2 & $120 \mathrm{ps}$ & $300 \mathrm{ps}$ & $100 \mathrm{KHz}$ \\
\hline
\end{tabular}

vided a fixed $f_{\text {rep }}$ of $100 \mathrm{kHz}$. Both pulses show in spite of different pulse shapes similar behavior in the frequency domain as shown in Fig. 3. Both pulse generators amplitudes are adjustable. Additionally linear attenuators where used to get a coarse predivision.

All presented measurements have been carried out with microcontrollers of the type AT90S8515 from Atmel. This device is a 40-pin 8-bit RISC microcontroller with 512 Bytes SRAM and 512 Bytes EEPROM. It is equipped with $32 \mathrm{I} / \mathrm{O}$ pins which are organized in four 8-bit I/O ports.

\section{Measurement results}

Based on the possibilities of the measurement setup four measurements where carried out by exposing different pins of the microcontroller to pulses with three different repetition rates. Fig. 4 shows the breakdown behavior of the different pins with increasing pulse amplitude. All configurations show basically the same behavior with increasing amplitude. Noticeable is the difference between the thresholds of the several setups, a fact that has been observed also during single pulse investigations (Camp, 2004). The susceptibility of the electronic device is highly dependable on the function of the exposed pins. Furthermore this measurement shows a general decrease of the thresholds with increasing repetition rate.

The breakdown values of the tested microcontrollers for the different measurement configurations are presented in Fig. 5. The decrease of the breakdown value with increasing repetition rate of every single microcontroller is clearly observable. Furthermore the variation of the breakdown value between the several devices decreases with rising repetition rate. This variation is described with the Breakdown Band- 

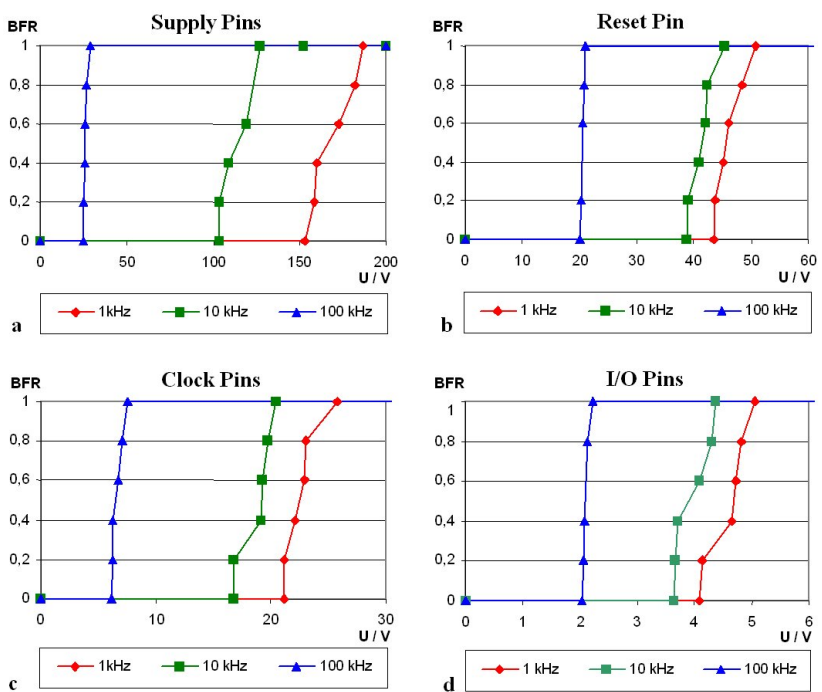

d $\rightarrow-1 \mathrm{kHz} \rightarrow-10 \mathrm{kHz} \rightarrow 100 \mathrm{kHz}$

Fig. 4. BFR: (a) Supply Pins; (b) Reset Pin; (c) Clock Pins; (d) I/O Pins.

width (BB) as mentioned above. In order to demonstrate the described effects more clearly Fig. 6a shows the breakdown thresholds extracted from the appropriate Weibull Distribution while the breakdown bandwidths are presented in Fig. 6b. From both diagrams we can directly extract the information that BT as well as BB are decreasing dramatically with rising pulse repetition rate. Remarkable is that in the worst case (I/O pins) the value of the breakdown threshold at $f_{\text {rep }}=100 \mathrm{kHz}, B T(\mathrm{I} / \mathrm{O}, 100 \mathrm{kHz})=2,01 \mathrm{~V}$, is less than half the value of the supply voltage and the high level of the microcontroller $\left(U_{C C}=U_{\text {High }}=5 \mathrm{~V}\right)$. With further increase of the repetition rate in the future a noteworthy continue of the decrease of the breakdown thresholds is unlikely. For $5 \mathrm{~V}$ logic devices the difference between lowest value for high-level and highest value for low-level $U_{H, \min }-U_{L, \max }=3 \mathrm{~V}-1.5 \mathrm{~V}=1.5 \mathrm{~V}$. The minimum voltage needed to switch a bit from one state to another is nearly reached.

Two characteristic values where defined in order to describe the variation of the breakdown behavior. For an arbitrary ratio of repetition rates $\mathrm{A} / \mathrm{B}$ we define the value for Threshold Variation (TV) as follows:

$T V(A / B)=\frac{B T_{A}}{B T_{B}}, \quad$ with $\quad A>B$

The definition of the Breakdown Variation (BV) in Eq. (4) is similar.

$B V(A / B)=\frac{B B_{A}}{B B_{B}}, \quad$ with $\quad A>B$

Table 2 shows both values at different ratios of the repetition rates. The TV decreases with increasing repetition rate. At $f_{\text {rep }}=10 \mathrm{kHz} T V(10 / 1)=86 \%$ of the pulse amplitude at
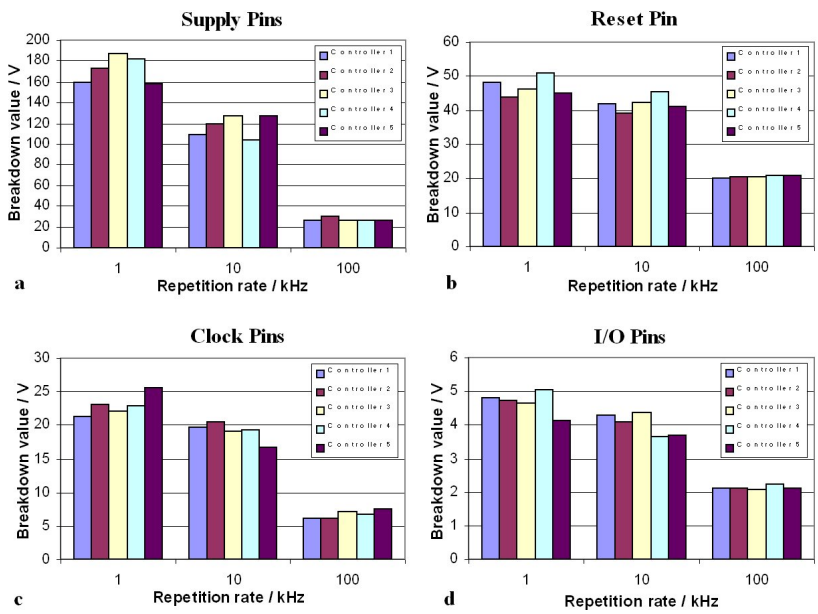

Fig. 5. Breakdown values: (a) Supply Pins; (b) Reset Pin; (c) Clock Pins; (d) I/O Pins.
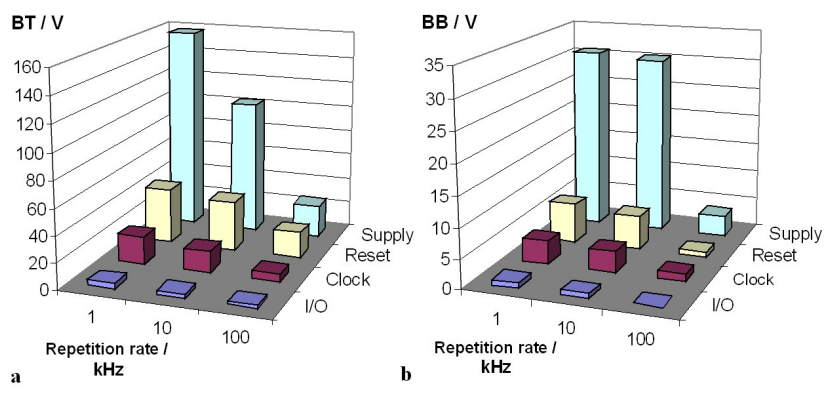

Fig. 6. Breakdown Thresholds (BT) and Breakdown Bandwidths (BB) of the different configurations with varying pulse repetition rate.

$f_{\text {rep }}=1 \mathrm{kHz}$ is needed for breakdown during I/O pins exposure. At ten times higher frequencies only $T V(100 / 10)=53 \%$ of the amplitude at $f_{\text {rep }}=10 \mathrm{kHz}$ is necessary to gain breakdowns. If we compare minimum and maximum frequencies less than one half $(T V(100 / 1)=46 \%)$ of the former amplitude is required. In the worst case (supply pins exposure) the $T V(100 / 1)$ value is only $16 \%$.

The BV values also decrease with rising repetition rate. While during $\mathrm{I} / \mathrm{O}$ pins exposure at $f_{\text {rep }}=10 \mathrm{kHz}$ $B V(10 / 1)=80 \%$ is required for breakdown of all microcontrollers, only $B V(100 / 10)=22 \%$ is necessary at ten times higher frequencies. In comparison of minimum and maximum frequencies $B V(100 / 1)$ is only $18 \%$. In the worst case (supply and reset exposure) $B V(100 / 1)=12 \%$. Thus the possibility to hit is increased by $88 \%$.

\section{Summary}

After several investigations (Camp et al., 2002; Camp, 2004; Camp et al., 2004) concerning the susceptibility of electronic devices to single transients this article shows a systematic 
Table 2. Threshold Variation (TV) and Bandwidth Variation (BV) with increasing repetition rate.

\begin{tabular}{lcccccc}
\hline Setup & TV (10/1) & BV (10/1) & TV (100/10) & BV (100/10) & TV (100/1) & BV (100/1) \\
\hline Supply & 0,68 & 0,98 & 0,23 & 0,12 & 0,16 & 0,12 \\
\hline Reset & 0,9 & 0,88 & 0,49 & 0,14 & 0,44 & 0,12 \\
\hline Clock & 0,83 & 0,9 & 0,35 & 0,35 & 0,29 & 0,31 \\
\hline I/O & 0,86 & 0,8 & 0,53 & 0,22 & 0,46 & 0,18 \\
\hline
\end{tabular}

analysis of the breakdown behavior of microcontrollers exposed to repetitive pulses with varying repetition rate.

During this analysis it can be observed that the breakdown threshold is sinking with increasing repetition rate while the possibility to hit a certain microcontroller at a critical system state is higher. Therefore the electronic device is overall more susceptible to pulses if applied with higher repetition rates. Similar to the results of single pulse investigations it can be stated that all the observed effects are correlated with the exposed pins functions. The I/O pins of the investigated microcontrollers are the most susceptible pins while the supply pins are the least susceptible ones.

After definition of two characteristic values it is shown that in the worst case the breakdown threshold can be lowered to only $16 \%$ of the former amplitude by increasing the repetition rate from $1 \mathrm{KHz}$ to $100 \mathrm{kHz}$. Remarkable is in this context the fact that the necessary breakdown threshold drops under less than one half of the supply voltage. A further noteworthy decrease under the value of $2 \mathrm{~V}$ is unlikely based on the electrical characteristics of the devices. The possibility to hit the device in a risky system state is increased due to the same repetition rate variation by up to $88 \%$. Overall the increase of the repetition rate of applied pulses to an electronic device is a pretty good possibility to increase the ability to disturb the system and is only limited by the electrical characteristics of the electronics.
Acknowledgements. This investigation is part of the study "Protection of Electronic Systems against Electromagnetic Sources", commissioned by the Armed Forces Scientific Institute for Protection Technologies - NBC-Protection (Munster, Germany). The responsibility for the contents is with the authors.

The authors would like to thank Dr. M. Jung, Rheinmetall Waffe Munition $\mathrm{GmbH}$ for supporting these measurements.

\section{References}

Camp, M.: Empfindlichkeit elektronischer Schaltungen gegen transiente elektromagnetische Feldimpulse, Diss., Univ. of Hanover, Shaker Verlag, 2004.

Camp, M., Garbe, H., and Nitsch, D.: Influence of the Technology on the Destruction Effects of Semiconductors by Impact of EMP and UWB Pulses, IEEE Intern. Symposium on Electrom. Compatibility, USA, Minneapolis, 87-92, 2002.

Camp, M., Korte, S., and Garbe, H.: Classification of the Destruction Effects in CMOS-Devices after Impact of Fast Transient Electromagnetic Pulses, EUROEM, $14^{\text {th }}$ High Power Electrom. Conf. (HPEM 14), $7^{\text {th }}$ Ultra-Wide-Band Short-Pulse Electrom. Conf. (UWB SP7), $7^{\text {th }}$ Unexploded Ordnance Detection and Range Remediation Conf. (UXO 7), Germany, 96, 2004. 